\title{
Os efeitos do Functional Fascial Taping (FFT) associado a fisioterapia convencional na funcionalidade do joelho: um estudo quase-experimental
}

\section{The effects of Functional Fascial Taping (FFT) associated with conventional physiotherapy on the knee functionality: a quasi-experimental study}

\author{
Soraia Suellen Silva dos Santos ${ }^{1}$ \\ Ana Paula Monteiro de Araújo 2 (b) \\ Maria Clara Raiol da Silva ${ }^{3}$ \\ Renata Amanajás de Melo $^{4}$ (1) \\ Lucieny da Silva Pontes 5 \\ Ana Julia Cunha Brito ${ }^{6}$ \\ Biatriz Araújo Cardoso Dias 7 (1) \\ George Alberto da Silva Dias ${ }^{8}$ (1)
}

\author{
1Universidade Federal do Pará (Belém). Pará, Brasil. soraiasuellenss@gmail.com \\ 2-5Universidade do Estado do Pará (Belém). Pará, Brasil. anapfisioaraujo@gmail.com, clararaiols@hotmail.com, rennamelo@uol.com.br, \\ lucienypontes@hotmail.com \\ ${ }^{6}$ Faculdade Estácio Nazaré (Belém). Pará, Brasil. anajuliabrito@hotmail.com \\ ${ }^{7}$ Centro Universitário Metropolitano da Amazônia (Belém).Pará, Brasil. biatrizac@yahoo.com.br \\ ${ }^{8}$ Autor para correspondência. Universidade do Estado do Pará (Belém). Pará, Brasil. georgealbertodias@yahoo.com.br
}

RESUMO | INTRODUÇÃO: O Functional Fascial Taping (FFT) é uma técnica de aplicação de bandagem rígida objetivando estimular o alinhamento estrutural das fibras de colágeno da fáscia muscular, desobstruindo a passagem dos fluidos assegurando assim sua função. OBJETIVO: Avaliar os efeitos do FFT associado a Fisioterapia convencional nas disfunções crônicas no joelho baseado nas variáveis intensidade da dor, amplitude de movimento, força e flexibilidade muscular. METODOLOGIA: Tratou-se de um estudo quase-experimental realizado com 10 pacientes de ambos os gêneros e sintomatologia de dor e limitação funcional, estando em tratamento fisioterapêutico. Todos os pacientes foram submetidos a avaliação cinético-funcional, incluindo dados de dor, amplitude de movimento, força e flexibilidade muscular. A aplicação do FFT foi padronizada, onde primeiro identificou-se os vetores, seguido da aplicação do tape hipoalergênico. Depois aplicou-se três tiras de bandagem rígida, então, recobriu-se com o tape hipoalergênico novamente. A aplicação da bandagem ocorreu três vezes por semana, totalizando 10 sessões. RESULTADOS: Houve redução nos níveis de dor de mediana $10,0 \mathrm{~cm}$ para $2,0 \mathrm{~cm}$ ao final das 10 sessões $(Z=2,6 ; p=0,007)$. Aumentou a força muscular para extensão do joelho com mediana inicial de 4,0 para final de 4,0 ( $Z=2,0 ; p=0,04)$. A amplitude de movimento para flexão (Mediana: de $102,5^{\circ}$ para $110,0^{\circ}, Z=2,3, p=0,01$ ) e flexibilidade muscular (Mediana: de 5,0 cm para 0,0 cm, Z=2,0, $p=0,04$ ) também melhorou significavamente. CONCLUSÃO: O FFT pode ser utilizado como coadjuvante no tratamento de desordens no joelho, tanto ortopédicas quanto reumáticas, auxiliando a manutenção de uma mecânica funcional equilibrada, o que favorece a redução no quadro álgico, aumento no arco de movimento e melhora na força e flexibilidade muscular.

PALAVRAS-CHAVE: Fáscia. Joelho. Amplitude de movimento articular. Fisioterapia.
ABSTRACT | INTRODUCTION: Functional Fascial Taping (FFT) is a rigid bandage technique aimed at stimulating the structural alignment of the collagen fibers of the muscular fascia, unblocking the passage of fluids thus ensuring its function. OBJECTIVE: To evaluate the effects of FFT associated with conventional physiotherapy on chronic knee dysfunctions based on the variables pain intensity, range of motion, muscle strength and flexibility. METHODOLOGY: This is a quasiexperimental study performed with 10 patients of both genders and symptomatology of pain and functional limitation, being in physiotherapeutic treatment. All of them underwent kinetic-functional evaluation, including data on pain, range of motion, muscle strength and flexibility. The application was standardized: first we identified the vectors, followed by the application of hypoallergenic tape. Then applied three strips of rigid bandage, then covered or hypoallergenic tape again. One application three times a week, totaling 10 sessions. RESULTS: There was a reduction in pain levels from a median $10.0 \mathrm{~cm}$ to $2.0 \mathrm{~cm}$ at the end of the 10 sessions $(Z=2.6 ; p=0.007)$. Increased muscle strength for knee extension with initial median of 4.0 to final of $4.0(Z=2.0 ; p=0.04)$. Range of motion also improved significantly for knee flexion (Median: from $102.5^{\circ}$ to $110.0^{\circ}, Z=2.3, p=0.01$ ) and increased flexibility (Median: from $5.0 \mathrm{~cm}$ to $0.0, Z=2.0 \mathrm{~cm}, \mathrm{p}=0.04$ ). CONCLUSION: FFT can be used as a coadjuvant in the treatment of knee disorders, both orthopedic and rheumatic, allowing stimuli to maintain a more balanced functional mechanics, which favors the reduction in pain, an increase in the range of motion and improvement in strength and muscle flexibility. 


\section{Introdução}

As desordens ortopédicas e reumatológicas no joeIho se caracterizam por lesões de grande incidência multifatorial que desencadeiam processos inflamatórios resultando em síndromes dolorosas ${ }^{1}$. Muitas destas lesões são decorrentes da instabilidade articular, onde o joelho depende de fatores como a geometria, restrição de tecidos moles, a ação da musculatura e as cargas aplicadas pelo corpo para poder manter sua função? .

Em contrapartida, quando uma articulação sinovial é lesionada observa-se uma cadeia de comprometimentos entre seus elementos constituintes gerando alterações morfofuncionais que aumentam o estresse no segmento durante a execução de atividades físicas diárias, além de causar instabilidade mecânica e dor na qual afeta a capacidade funcional do indivíduo ${ }^{3,4}$.

Nas repercussões osteomusculares destaca-se a fraqueza da musculatura do quadríceps, causada por alterações sensoriais dos mecanorreceptores da articulação reduzindo, assim, a habilidade de ativação do músculo, e, consequentemente, redução da capacidade funcional predispondo a danos estruturais ${ }^{5}$.

Dentre os componentes anatômicos do joelho, tem-se a fáscia muscular que é uma estrutura periarticular composta de tecido conjuntivo fibroso rico em colágeno e elastina que ao ser submetida a sobrecargas mecânicas alteram sua composição ${ }^{6}$. Os estresses biomecânicos bruscos nesta articulação levam a ruptura do colágeno, e em uma tentativa de cicatrização do tecido fascial, ocorre a deposição desorganizada de colágeno resultando em fibrose, podendo afetar a circulação linfática, o desempenho muscular e a amplitude de movimento?

Vários métodos são utilizados na fisioterapia na tentativa de aceleração da síntese de colágeno e deposição de forma organizada. Uma nova alternativa empregada como intervenção terapêutica é o Functional Fascial Taping (FFT), que consiste em uma técnica não invasiva de aplicação de bandagem rígida cujo objetivo é estimular o alinhamento estrutural das fibras de colágeno da fáscia muscular, desobstruindo a passagem dos fluidos assegurando assim sua função. A manutenção desse estímulo na fáscia muscular induz um processo de reabilitação acelerado, visto que a transmissão dos impulsos de tensão permite que os músculos recobertos por este envelope conjuntivo garantam a capacidade de sustentação, contração e flexibilidade, auxiliando nas alavancas dos movimentos funcionais ${ }^{-}$.

Existe grande diversidade de recursos terapêuticos para o tratamento das disfunções no joelho. O FFT pode assumir um papel coadjuvante na associação desses recursos. Porém ainda há escassez de informações na literatura quanto ao uso desta bandagem, devido ser uma técnica ainda recente no campo da reabilitação. Um estudo voltado para analisar os efeitos do FFT em indivíduos com presença de dor e diminuição da função nos joelhos traria benefícios para a área acadêmica e profissional buscando melhora na recuperação destes pacientes. Diante disso, o presente estudo objetivou avaliar os efeitos do FFT associado a Fisioterapia convencional em pacientes com disfunções crônicas no joelho baseado nas variáveis intensidade da dor, amplitude de movimento, força e flexibilidade muscular.

\section{Metodologia}

\section{Amostra}

Este estudo foi submetido e aprovado pelo Comitê de Ética em Pesquisa Envolvendo Seres Humanos (CEP) do Instituto de Ciências da Saúde da Universidade Federal do Pará (UFPA) sob parecer número 813.807 (CAAE36573514.1.0000.0018), seguindo as normas da Resolução 466/12 do Conselho Nacional de Saúde. Tratou-se de um estudo do tipo quase-experimental, realizado na Clínica de Fisioterapia Desportiva Ortoclínica do Pará, em Belém do Pará, no período de outubro a novembro de 2014.

Participaram do estudo indivíduos de ambos os sexos, acima de 18 anos, selecionados por meio da amostragem não probabilística por conveniência, com diagnóstico clínico de lesão ortopédica e reumatológicas, pois os mesmos já se encontravam em tratamento fisioterapêutico. Este consistiu em recursos de eletroterapia de baixa intensidade como a estimulação elétrica nervosa transcutânea (TENS) (Largura de pulso: 75us e frequência: $100 \mathrm{~Hz}$, durante $20 \mathrm{mi}-$ nutos), seguido de cinesioterapia ativa livre para flexibilidade muscular e cinesioterapia resistida para ganho de força, bem como exercícios proprioceptivos para ganho de equilíbrio. 
Como critério de inclusão deveriam apresentar intensidade de dor entre moderada e intensa pela Escala Visual Analógica (EVA) e arco de movimento incompleto para flexão do joelho acometido. Já os critérios de exclusão consistiram de pacientes com amplitude de movimento com limitação fixa, quadros neoplásicos e reação alérgica ao material utilizado.

\section{Procedimentos de avaliação}

Antecedendo a aplicação da técnica realizou-se avaliação cinético-funcional, para coleta de dados pessoais e do exame físico. Na avaliação da dor foi utilizada a EVA que mensura a intensidade da dor, onde pontuações de 0 a 2 são classificadas como leve; 3 a 7, classificada como moderada; e 8 a 10, classificada como intensa9. A graduação da força muscular dos extensores e flexores do joelho foi mensurada por meio da escala do Medical Research Council (MRC) ${ }^{10}$, realizando a contração muscular concêntrica destes músculos estando o paciente sentado com o membro pendente. Esta escala consiste na graduação da força de 0 a 5, de acordo com a seguinte classificação: Grau 0 (Sem contração); Grau 1 (Contração muscular visível ou palpável sem movimentação); Grau 2 (Movimento ativo com eliminação da gravidade); Grau 3 (Movimento ativo contra a gravidade); Grau 4 (Movimento ativo contra gravidade e resistência) e Grau 5 (Força normal).

A mensuração da amplitude de movimento de flexão do joelho foi realizada utilizando o goniômetro (graus) grande da marca $\mathrm{CARCl}$, estando o paciente em decúbito dorsal sobre uma maca e o fisioterapeuta ao lado do membro a ser testado. O eixo do goniômetro estava localizado sobre a linha articular do joelho, com o braço fixo paralelo à superfície lateral do fêmur dirigido para o trocânter maior e com o braço móvel paralelo a face lateral da fíbula dirigido ao maléolo lateral, solicitou ao paciente a flexão voluntária da articulação, onde o ponto da mensuração era quando se sentia um leve desconforto ou retesamento muscular.

Para a flexibilidade muscular da cadeia posterior do membro inferior, utilizou-se o teste $3^{\circ}$ dedo-solo ${ }^{11}$ solicitando que o paciente permanecesse com os pés unidos e os joelhos extendidos durante a flexão anterior de tronco, na tentativa de tocar o solo. Através da fita métrica $(\mathrm{cm})$ foi possível medir a distância do $3^{\circ}$ dedo ao solo e assim graduar a flexibilidade muscular dos pacientes. Todas as variáveis estudadas foram reavaliadas ao final das 10 sessões do FFT.

\section{Aplicação do FFT}

A aplicação do FFT ocorreu utilizando a identificação dos vetores (Figura 1A). Primeiramente foi solicitado ao paciente a identificação do ponto de dor no joelho. Em seguida, a partir deste ponto, realizou-se a identificação dos vetores. O vetor é a direção da pressão digital que leva ao alívio da dor e, consequentemente, melhora dos movimentos do joelho. Assim, a aplicação da bandagem deu-se no sentido dos vetores encontrados com máxima redução da dor.

Figura 1. (A) Identificação dos vetores (seta preta). (B) Aplicação do tape hipoalergênico. (C) Aplicação das três tiras de tape para cada vetor encontrado. (D) Finalização da técnica com a aplicação do tape hipoalergênico

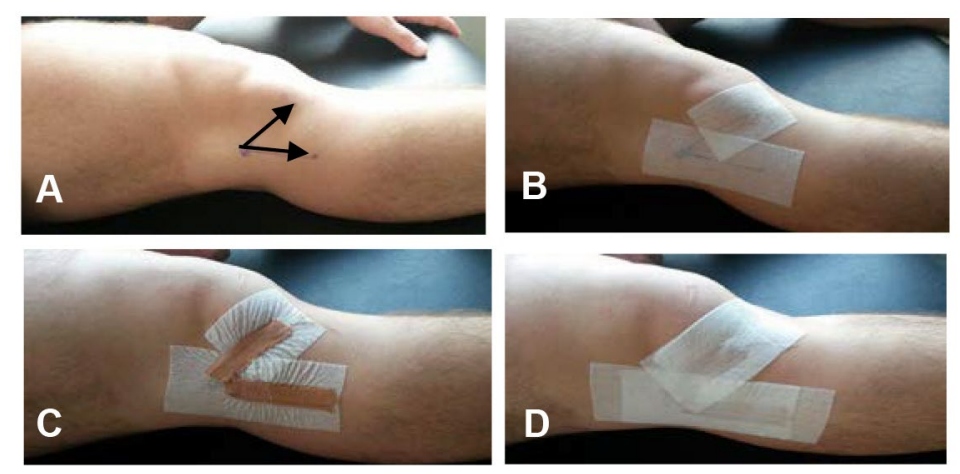


A aplicação da bandagem precedeu-se por um processo padronizado. Primeiro foi realizado a limpeza da pele, seguida de tricotomia quando necessário conforme pode ser observado na figura 1A. Após este processo, utilizou-se cola de contato (Coloplast Prep Protective Skin Barrier) para melhor fixação da bandagem, conforme orientado pela própria técnica. Em seguida foi aplicado o tape hipoalergênico (Figura 1B), no sentido dos vetores identificados, evitando qualquer irritação da pele posteriormente à aplicação do FFT, favorecendo a acomodação da bandagem rígida. Para a aplicação desta bandagem, o tape rígido foi aplicado consecutivamente após pequenas tensões na pele do paciente afim de causar tensão na fáscia muscular. E para obter este resultado foram aplicadas três tiras de tape sequenciais para cada vetor encontrado (Figura 1C). Para finalizar, recobriu-se a bandagem rígida com o tape hipoalergênico novamente (Figura 1D). Após a aplicação do FFT os pacientes participavam normalmente do tratamento de Fisioterapia. A proposta do estudo foi a aplicação deste recurso de forma alternada três vezes por semana, totalizando 10 sessões.

\section{Análise estatística}

Adotou-se o software Excel® 2010 para entrada dos dados e confecção das tabelas, bem como o software BioEstat 5.0 na análise estatística. As variáveis categóricas foram apresentadas como frequências e as numéricas por meio de medidas de tendência central e dispersão. Para a análise da significância dos dados utilizou-se o teste de Wilcoxon. Todos os resultados foram considerados estatisticamente significantes no nível de significância de $5 \%(p \leq 0,05)$.

\section{Resultados}

A amostra constituiu de 10 pacientes com mediana de 45,5 anos e intervalo interquartil de 24,5 anos, predominantemente composto pelo sexo feminino com $90 \%(n=9)$ (Tabela 1). Em dois pacientes não foi necessário aplicar o FFT até o final das 10 sessões, uma vez que a dor cessou entre a quarta e quinta sessão não sendo mais útil a sua aplicação. No entanto, estes continuaram com o tratamento proposto pela clínica e fizeram parte da avaliação final normalmente.

Tabela 1. Dados gerais dos pacientes atendidos com o FFT

\begin{tabular}{|c|c|c|c|}
\hline Variáveis & $\mathbf{N}$ & & $\%$ \\
\hline \multicolumn{4}{|l|}{ Idade (anos) } \\
\hline Mediana & & 45,5 anos & \\
\hline Intervalo interquartil & & 24,5 anos & \\
\hline \multicolumn{4}{|l|}{ Sexo } \\
\hline Masculino & 1 & & $10 \%$ \\
\hline Feminino & 9 & & $90 \%$ \\
\hline
\end{tabular}

Inicialmente a mediana da intensidade de dor encontrada foi de 10,0 sendo considerado dor intensa. Ao final das 10 sessões de Fisioterapia associando o FFT, a mediana de dor diminuiu para 2,0 (dor leve), com resultados significantes $(Z=2,6 ; p=0,007)$, observando melhora do quadro álgico nestes pacientes (Tabela 2$)$. 
Tabela 2. Variáveis analisadas antes e após aplicação do FFT

\begin{tabular}{|c|c|c|c|c|c|c|}
\hline \multirow{2}{*}{ Variáveis } & \multicolumn{2}{|c|}{ Avaliação Inicial } & \multicolumn{2}{|c|}{ Avaliação Final } & \multirow{2}{*}{$z$} & \multirow{2}{*}{ p-valor } \\
\hline & Mediana & IIQ & Mediana & IIQ & & \\
\hline Dor $(\mathrm{cm})$ & 10,0 & 4,0 & 2,0 & 4,0 & 2,6 & 0,007 * \\
\hline \multicolumn{7}{|l|}{ Força muscular } \\
\hline Flexão & 3,5 & 1,0 & 4,0 & 0,0 & 1,6 & 0,10 \\
\hline Extensão & 4,0 & 1,0 & 4,0 & 0,75 & 2,0 & $0,04 *$ \\
\hline \multicolumn{7}{|l|}{ Goniometria $\left({ }^{\circ}\right)$} \\
\hline Flexão & 102,5 & 15,0 & 110,0 & 8,75 & 2,3 & 0,01 * \\
\hline Teste $3^{\circ}$ dedo-solo $(\mathrm{cm})$ & 5,0 & 17,5 & 0,0 & 5,0 & 2,0 & $0,04 *$ \\
\hline
\end{tabular}

Intervalo interquartil (II Q). * Resultado estatisticamente significante, teste de Wilcoxon $(\mathrm{p} \leq 0,05)$.

Com relação a variável força muscular, para o movimento de flexão do joelho, não foi observado resultado significante $(Z=1,6 ; p=0,10)$. Inicialmente a mediana da força muscular era de 3,5 segundo a escala do MRC, e após as aplicações do FFT a mediana passou para 4,0 (Tabela 2). No entanto, para o movimento de extensão do joelho, estatisticamente houve ganho de força muscular $(Z=2,0 ; p=0,004)$, com mediana inicial de 4,0 e mediana final de 4,0 após o FFT, conforme observado na tabela 2 .

Quanto à variável amplitude de movimento para flexão do joelho, foi observado resultado estatisticamente significante. A mediana inicial da amplitude articular era de $102,5^{\circ}$ e a mediana final foi de $110,0^{\circ}$ $(Z=2,3 ; p=0,01)$, conforme mostra a tabela 2. E por fim, com relação a flexibilidade muscular da cadeia posterior do membro inferior, inicialmente foi observado mediana de $5,0 \mathrm{~cm}$ e ao final das sessões com mediana de $0,0 \mathrm{~cm}$ apresentando resultados significativos $(Z=2,0 ; p=0,04)$.

\section{Discussão}

O FFT é uma proposta terapêutica de melhora da funcionalidade e permitir que a reabilitação comece em um ambiente com menos queixas álgicas, proporcionando desta forma uma janela de oportunidade para uma intervenção terapêutica mais acessível e eficiente ao paciente $\mathrm{e}^{12,13}$.

No contexto da reabilitação fisioterapêutica, o FFT, apesar da diversidade de tratamento, no que diz respeito às disfunções no joelho, assume um papel coadjuvante na associação desses tratamentos, haja vista seus diversos benefícios para a sintomatologia observada nessas disfunções ${ }^{8,13}$. Neste estudo foi possível observar, de forma significativa, diminuição do quadro álgico nos pacientes pesquisados. A mensuração álgica de característica intensa passou a ser leve após 10 sessões de aplicação do FFT, ainda que associado a outros recursos da fisioterapia, pois mesmo em processo de reabilitação ao fazer uso do recurso proposto observou-se a aceleração na resposta ao tratamento.

Este achado pode ser comprovado por estudos que utilizaram este mesmo método e também obtiveram redução da dor devido ao fato deste, facilitar o deslizamento entre a fáscia e o músculo em decorrência da liberação de colágeno ${ }^{8,14}$. Spina, Cameron e Alexander ${ }^{15}$ encontraram, em seu estudo de caso, resultados na melhora na dor após a terceira sessão do FFT na região do antepé em paciente com neuroma de Morton. Seu estudo teve como enfoque dados referentes ao alívio de dor e funcionalidade, mensurados por meio da EVA e a escala de funcionalidade Functional Foot Index (FFI). Conforme consta no presente estudo, a EVA é bastante utilizada como ferramenta de avaliação das queixas álgicas.

Além disso, ratificando este achado dois pacientes não precisaram completar o número de aplicações do FFT estipuladas, já que a dor havia cessado na quarta sessão. Vale ressaltar ainda que em todos os casos, os pacientes relataram diminuição do quadro álgico na primeira aplicação da bandagem. Corroborando com resultados de um estudo desenvolvido por Chen, Alexander e Cook $^{16}$ os quais demonstraram que pacientes com dor lombar não específica obtiveram melhora significativa na queixa álgica após aplicação do FFT. 
Quanto à força muscular, ainda não há estudos que confirmem relação direta entre o aumento da força muscular com a aplicação do FFT. Porém, neste estudo observou-se ganho somente para os extensores de joelho de forma significativa, de acordo com a escala do MRC. Acredita-se, com este resultado, que a relação existente entre a bandagem e o ganho força muscular possa estar atrelada ao fato de que com o alívio da dor o paciente seja capaz de realizar maior movimento articular através da diminuição de carga sobre a articulação, e, assim, ter melhor rendimento muscular durante a execução dos exercícios ${ }^{17}$.

Em relação a amplitude de movimento houve ganho significativo para flexão do joelho com aumento de 9,5 após as aplicações do FFT. Reforçando os resultados de Spina, Cameron e Alexander ${ }^{15}$, que também observaram ganho na amplitude de movimento e que pode estar relacionado a melhora dos níveis de dor, semelhante ao que foi observado no presente estudo. Em um apanhado de pesquisas acerca dos resultados obtidos através do uso do FFT, Alexander 13 afirma que em mais de $95 \%$ dos casos é observado melhora significante na amplitude de movimento. Segundo o autor, isso é possível, pois há o deslocamento e liberação dos pontos de fibrose desenvolvidos nas fáscias pelo processo inflamatório. Essa liberação facilita o processo de deslizamento entre planos e tecidos, como acontece entre a fáscia muscular e o músculo propriamente dito, facilitando a resposta de contração muscular e consequente meIhora do arco de movimento.

Em resposta a lesão, a literatura afirma que o corpo estimula a produção de colágeno ${ }^{18}$. A aplicação do método FFT promove alongamento contínuo desse colágeno e influencia tanto na estrutura quanto na função do tecido fascial, facilitando o deslizamento entre as camadas fasciais ${ }^{19}$. A frequente redução da dor e melhora da amplitude de movimento, pode ser explicado pela alteração da atividade nociceptiva aferente dos mecanorreceptores ${ }^{20,21}$ causada pela aplicação da bandagem. Assim, o que diferencia esta técnica das demais é a especificidade, a direção e a quantidade do tape aplicado ${ }^{12}$.

Em relação a flexibilidade muscular da cadeia posterior do membro inferior, também foi obtido resultado significante para o teste do $3^{\circ}$ dedo-solo. Isso demonstra que a fáscia muscular pode interferir no funcionamento da mecânica articular do joelho e no contexto global da cadeia muscular posterior.
Este resultado pode ser sustentado por meio da explicação da ação do FFT na arquitetura fascial ${ }^{12}$, permitindo um melhor deslizamento dos componentes musculares, auxiliando seu alongamento. Porém, semelhante à força muscular, não há dados que confirmem relação direta entre bandagens e o aumento no alongamento muscular, mas sim na melhora da flexibilidade entre o deslizamento da fáscia e músculo.

Muito se escreve a respeito de outro tipo de bandagem, conhecida como o Kinesio Taping (KT), dentro de um protocolo de reabilitação para esportistas 22,23 , e esta técnica não se restringe somente a este público. $\mathrm{Na}$ literatura se encontra vários estudos em diversas situações clínicas. Pouco ainda se escreve sobre o FFT, o que torna difícil o embasamento científico para o desenvolvimento de estudos envolvendo esta bandagem. Mas apesar deste obstáculo, uma pesquisa voltada para a análise da técnica em pacientes com presença de dor e diminuição na função dos joelhos traria benefícios para a área acadêmica e profissional que visa à qualidade de vida destes pacientes.

Este estudo apresenta algumas limitações como não apresentar randomização e não haver um grupo controle, assim como possuir um viés de seleção, e limitações quanto a validade interna e externa.

\section{Conclusão}

A pesquisa mostrou redução na intensidade da dor, melhora da amplitude de movimento para extensão do joelho, ganho de força muscular e aumento da flexibilidade muscular quando associado o FFT a Fisioterapia convencional. Mostrando neste estudo que esta técnica pode ser bastante útil na reabilitação destes pacientes. Porém, é necessário novos estudos que verifiquem a atuação da bandagem na tentativa de fornecer novas informações fisiológicas na utilização desta técnica.

\section{Contribuições dos autores}

Santos SSS participou da concepção, delineamento, coleta dos dados e interpretação dos resultados. Araújo APM, Silva MCR participaram da busca de dados da literatura, interpretação dos dados e redação do artigo. Melo RA participou da concepção do projeto e revisão crítica do artigo. Pontes LS participou da concepção, delineamento e revisão do artigo. Brito AJC participou do delineamento do estudo, busca de dados na literatura e análise dos dados. 
Cardoso-Dias BA participou da concepção, delineamento, análise estatística dos dados da pesquisa, interpretação dos resultados. Dias GAS participou da concepção, análise estatística dos dados da pesquisa, interpretação dos resultados e redação.

\section{Conflitos de interesses}

Nenhum conflito financeiro, legal ou político envolvendo terceiros (governo, empresas e fundações privadas, etc.) foi declarado para nenhum aspecto do trabalho submetido (incluindo, mas não se limitando a subvenções e financiamentos, participação em conselho consultivo, desenho de estudo, preparação de manuscrito, análise estatística, etc.).

\section{Referências}

1. Fitzgerald GK, Piva SR, Irrgang J, Bouzubar F, Starz TW. Quadriceps activation failure as a moderator of the relationship between quadriceps strength and physical function in individuals with knee osteoarthritis. Arthritis Rheum. 2004;51(1):40-8. doi: 10.1002/art.20084

2. Williams GN, Chmielwski T, Rudolph K, Buchanant TS, SnyderMackler L. Dynamic knee stability: current theory and implications for clinicians and scientists. J Orthop Sports Phys Ther. 2001;31(10):546-66. doi: 10.2519/jospt.2001.31.10.546

3. Vasconcelos KSS, Dias JMD, Dias RC. Relação entre intensidade de dor e capacidade funcional em indivíduos obesos com osteoartrite de joelho. Rev Bras Fisioter. 2006;10(2):213-8.

4. Clark NC. Predictors of Knee Functional Joint Stability in Uninjured Physically Active Adults [dissertation]. Reino Unido: School of Health and Rehabilitation Sciences: University of Pittsburgh; 2014.

5. Nogueira JFS, Lins CAA, Souza AVC, Brasileiro JS. Efeitos do aquecimento e do alongamento na resposta neuromuscular dos isquiotibiais. Rev Bras Med Esporte. 2014;20(4):262-266. doi: 10.1590/1517-86922014200401387

6. Grossi JVM. Estudo do colágeno do tipo I e tipo III na linha alba de pacientes obesos mórbidos [dissertação]. Rio Grande do Sul: Universidade Federal do Rio Grande do Sul; 2016.

7. Aquino CF, Viana SO, Fonseca ST. Comportamento biomecânico e resposta dos tecidos biológicos ao estresse e à imobilização. Fisioter Mov. 2005;18(2):35-43.

8. Alexander R. Functional Fascial Taping. SportEX dynamics. 2007;12(4):10-13.

9. Myles PS, Myles DB, Galagher W, Boyd D, Chew C, MacDonald N et al. Measuring acute postoperative pain using the visual analog scale: the minimal clinically important difference and patient acceptable symptom state. Br J Anaesth. 2017;118(3):424-9. doi: 10.1093/bja/aew466
10. Latronico N, Gosselink R. Abordagem dirigida para o diagnóstico de fraqueza muscular grave na unidade de terapia intensiva. Rev Bras Ter Intensiva. 2015;27(3):199-201. doi: $\underline{10.5935 / 0103-507 X .20150036}$

11. Perret C, Poiraudeau S, Fermanian J, Colau MM, Benhamou MA, Revel M. Validity, reliability, and responsiveness of the fingertip-to-floor test. Arch Phys Med Rehabil. 2001;82(11):156670. doi: 10.1053/apmr.2001.26064

12. Alexander R. Functional Fascial Taping Research. Terra Rosa e-magazine. 2013;13:15-8.

13. Alexander R. Functional Fascial Taping and Research. Terra Rosa e-magazine. 2014;1(16):24-8.

14. Alexander R. Functional fascial taping for lower back pain: a case report. J Body Mov Ther. 2008;12(3):263-4. doi: 10.1016/j. jbmt.2008.04.019

15. Spina R, Cameron M, Alexander R. The effect of Functional Fascial Taping on Morton's Neuroma. Australas Chiropr Osteopathy. 2002;10(1):45-50.

16. Chen SM, Alexander R, Lo SK, Cook J. Effects of Funcional Fascial Taping on pain and function in patients with non-especific low back pain: a pilot randomized controlled Trial. Clin Rehabil. 2012;26(10):924-33. doi: 10.1177/0269215512441484

17. Perrin DH. Bandagens Funcionais e Órteses Esportivas. Porto Alegre: Artmed; 2012.

18. Barroso GC, Thiele ES. Lesão muscular nos atletas. Rev Bras Ortop. 2011;46(4):354-58. doi: 10.1590/S0102$\underline{36162011000400002}$

19. Schleip R, Gabbiani G, Wilke J, Naylor I, Hinz B, Zorn A et al. Fascia is able to actively contract and may thereby influence musculoskeletal dynamics: a histochemical and mechanographic investigation. Front Physiol. 2019;10:1-15. doi: $10.3389 /$ fphys.2019.00336

20. Alves Neto O. Dor: princípios e prática. Porto Alegre: Artmed; 2009.

21. Ay S, Konak HE, Evcik D, Kibar S. Efetividade do kinesio taping na dor e incapacidade na síndrome dolorosa miofascial cervical. Rev Bras Reumatol. 2017;57(2):93-99. doi: 10.1016/j. $\underline{\text { rbr.2015.12.004 }}$

22. Kaya E, Zinnuroglu M, Tugcu I. Kinesio taping compared to physical therapy modalities for the treatment of shoulder impingement syndrome. Clin Rheumatol. 2011;30(2):201-7. doi: 10.1007/s10067-010-1475-6

23. Luz Júnior MA, Sousa MV, Neves LAFS, Cezar AAC, Costa LOP. Kinesio Taping ${ }^{\circledR}$ is not better than placebo in reducing pain and disability in patients with chronic non-specific low back pain: a randomized controlled trial. Braz J Phys Ther. 2015;19(6):482-90. doi: 10.1590/bjpt-rbf.2014.0128 